\title{
Atomic scale dynamics drive brain-like avalanches in percolating nanostructured networks
}

Matthew D. Pike, ${ }^{\dagger} \S$ Saurabh K. Bose, ${ }^{\ddagger}, \S$ Joshua B. Mallinson, ${ }^{\ddagger}$ Susant K.

Acharya ${ }^{\ddagger}$ Shota Shirai ${ }^{\ddagger}$ Edoardo Galli, ${ }^{\ddagger}$ Stephen J. Weddell, ${ }^{\dagger}$ Philip J. Bones, ${ }^{\dagger}$ Matthew D. Arnold, " and Simon A. Brown $*,+$

$\dagger$ Electrical and Computer Engineering, University of Canterbury, Private Bag 4800, Christchurch 8140, New Zealand

$\ddagger$ The MacDiarmid Institute for Advanced Materials and Nanotechnology, School of Physical and Chemical Sciences, Te Kura Matū, University of Canterbury, Private Bag 4800,

Christchurch 8140, New Zealand

ISchool of Mathematical and Physical Sciences, University of Technology Sydney, Australia $\S$ These authors made equal contributions.

E-mail: simon.brown@canterbury.ac.nz 


\section{${ }_{15}$ Keywords}

\begin{abstract}
Self-assembled networks of nanoparticles and nanowires have recently emerged as promising systems for brain-like computation. Here we focus on percolating networks of nanoparticles which exhibit brain-like dynamics. We use a combination of experiments and simulations to show that the brain-like network dynamics emerge from atomic-scale switching dynamics inside tunnel gaps that are distributed throughout the network. The atomic-scale dynamics emulate leaky integrate and fire (LIF) mechanisms in biological neurons leading to the generation of critical avalanches of signals. These avalanches are quantitatively the same as those observed in cortical tissue and are signatures of the correlations that are required for computation. We show that the avalanches are associated with dynamical restructuring of the networks which selftune to balanced states consistent with self-organised criticality. Our simulations allow

visualisation of the network states and detailed mechanisms of signal propagation.
\end{abstract}

Brain-like networks, percolation, scale-free dynamics, long-range temporal correlations, criticality, nanoparticle networks, neuromorphic computing 
Neuromorphic, or brain-like, computing is motivated both by the recognition that traditional integrated circuit technologies are reaching fundamental limits, ${ }^{1,2}$ and by the remarkable capability of the biological brain to perform tasks such as pattern recognition in an extremely energy-efficient way. ${ }^{3-5} \mathrm{~A}$ wide variety of brain-inspired approaches to computing are being investigated, using for example CMOS neurons and synapses, ${ }^{6,7}$ memristors, ${ }^{8-10}$ atomic switches, ${ }^{11,12}$ and phase change materials, ${ }^{13}$ but there have been relatively few attempts to develop intrinsically brain-like architectures which might support neuromorphic computing in a more natural way than standard (highly-organised) chip architectures.

Motivated by calculations which show that optimal information processing is achieved by intrinsically complex architectures operating at criticality ${ }^{14,15}$ and that scale-free, hierarchical networks are valuable ${ }^{16}$ in enhancing neuromorphic approaches such as reservoir computing (RC), ${ }^{5,17,18}$ several groups have begun to explore the properties of self-assembled nanoscale networks. ${ }^{19,20}$ This approach was initially driven by investigations of networks of silver nanowires, ${ }^{12,21}$ which exhibit interesting dynamics and were used in first attempts to perform waveform regression tasks. ${ }^{22}$ More recently other nanowire systems have been investigated ${ }^{23,24}$ and it has emerged that percolating-tunneling networks of nanoparticles also exhibit complex dynamics, ${ }^{25-28}$ brain-like avalanches and criticality ${ }^{29}$ and long-range temporal correlations (LRTCs) due to their intrinsically scale-free network architectures. ${ }^{30}$

In this Letter we show that brain-like network dynamics in percolating networks of nanoparticles emerge from atomic scale dynamics inside tunnel junctions within the networks. We first present experimental data that reveals the atomic scale dynamics and show that they emulate some of the functions of biological neurons. We then use computer simulations to demonstrate that, when coupled with the underlying scale-free network architecture ${ }^{30}$ these dynamics lead to critical avalanches of signals that are similar to those observed experimentally, and which in turn are quantitatively the same as those observed in cortical tissue. ${ }^{15,31}$ We show that criticality emerges only in a parameter range where the network self-tunes to a state with an optimal number of pathways through the network, consistent 

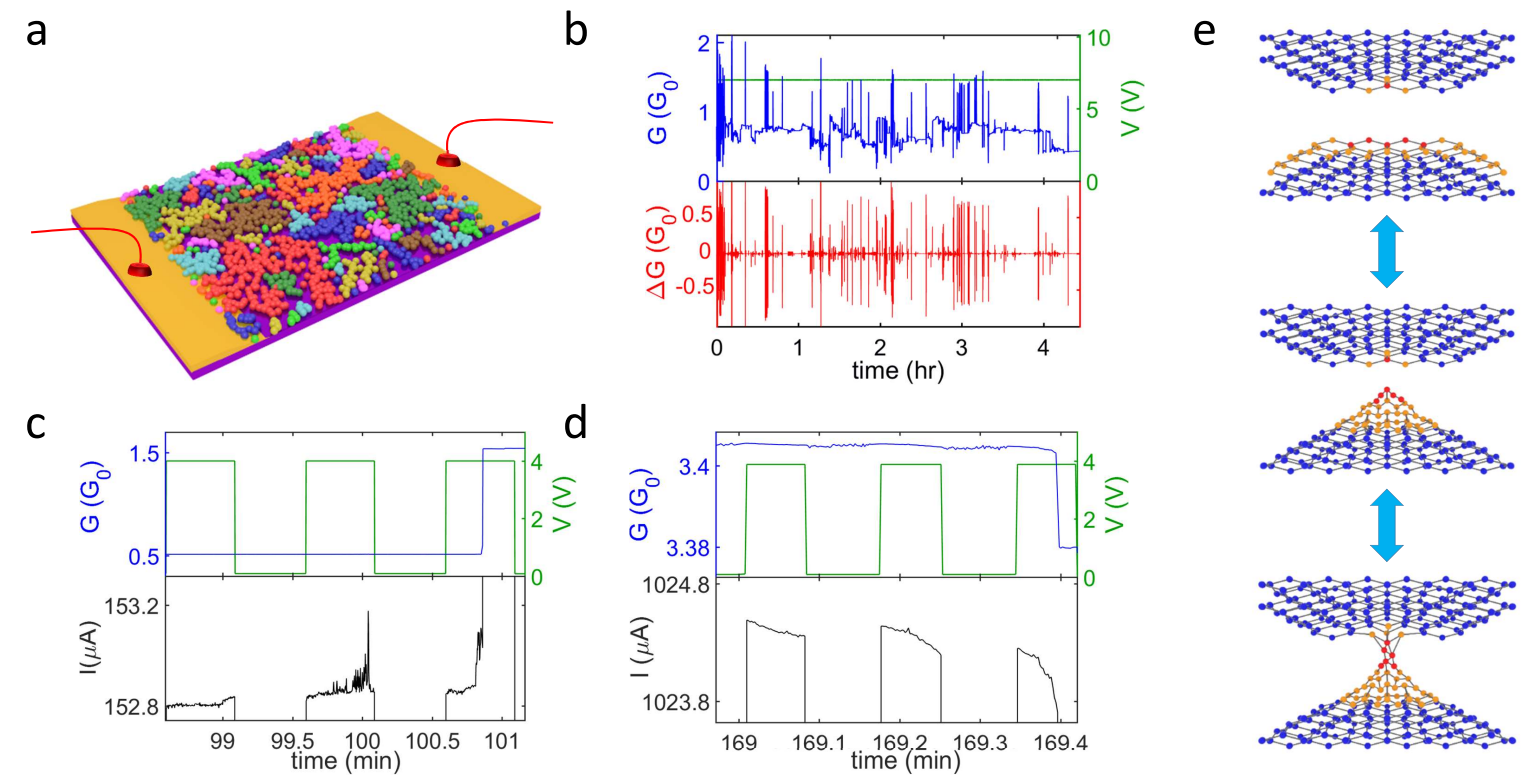

Figure 1: Atomic-scale dynamics in percolating nanoparticle networks. (a) Schematic illustrating two-terminal device geometry with the interconnected nanoparticle groups (different colours) separated via tunnel gaps. (b) Representative conductance data, measured over many hours with $100 \mathrm{~ms}$ sampling interval (see Methods), showing complex patterns of switching events and bursty dynamics. ${ }^{29,30}$ Voltage stimulus (green), device conductance $(G$, blue, in units of $G_{0}=2 \mathrm{e}^{2} / \mathrm{h}$, the quantum of conductance), and event size $(\Delta G$, red). (c) Low-voltage pulsed stimulus, focusing on a single switching event (time-window 2.5 mins), reveals clear signatures of signal integration prior to 'firing', corresponding to electric field induced atomic hillock formation. (d) Electromigration-induced reverse process reduces the average width of a pre-existing atomic filament (decreasing $I$ ), eventually resulting in filament-breaking. (e) Schematic of atomic-filament formation/destruction process. ${ }^{33-35}$

with self-organised criticality. ${ }^{32}$ Finally, by comparing the experimental and simulational results, we show that the distribution of measured changes in conductance reflects the dynamical structure of the network, and for the first time demonstrate the detailed mechanism for the propagation of critical avalanches in self-assembled networks.

Our percolating networks of nanoparticles are formed through deposition of nanoparti- 
cles onto silicon nitride substrates. ${ }^{25,26}$ Deposition is terminated when the fraction $(p)$ of the surface area covered with conducting particles approaches the percolation threshold $\left(p_{c} \sim\right.$ $68 \%$ ), which is a critical value separating the insulating and the conducting states. ${ }^{36}$ Figure 1a shows a schematic of our two electrode devices: during deposition (see Methods), particles come into contact and form interconnected groups which are separated by tunnel gaps (which have a distribution of sizes ${ }^{37}$ ), and which have varying sizes and fractal geometries. ${ }^{30}$ Groups are collections of particles that are in Ohmic contact with one another. We emphasise that after deposition the overall structure of the network is fixed, in contrast to many other devices (see e.g. Ref. 38) where memristive behaviour results from significant re-arrangements of nanoparticles. This distinction is illustrated in Figure S6 and Figure S7.

The tunnel gaps act as switching sites: upon application of an external voltage stimulus, atomic scale filaments can be formed (and subsequently broken) in the tunnel gaps, ${ }^{25}$ resulting in changes in the network conductance $(G)$ shown in Figure 1b. These switching events occur in bursts, or avalanches, that have been shown to exhibit ${ }^{29}$ the same statistical properties as avalanches of neuronal signals in the cortex,${ }^{31}$ thus demonstrating the strong spatio-temporal correlations required for $\mathrm{RC}$ and strong potential for optimal information processing ${ }^{39}$ - see Refs. 29,30 for further details.

The atomic scale switching processes that cause correlations and avalanches are yet to be studied in percolating networks, because most switching events occur on timescales ${ }^{11}$ that are far quicker than can be recorded by the measurement system. Here, using low-voltage pulsed stimulation, we have interrogated the switching processes and resolved for the first time the dynamics of some switching events. Figure 1c,d shows portions of experimental conductance traces, which capture formation (Figure 1c) and destruction (Figure 1d) of atomic scale filaments, following the behaviour shown schematically in Figure 1e and described in more detail in the next paragraph.

Figure 1c shows an initial increase in conductance as Electric Field Induced Surface Diffusion (EFISD) ${ }^{33,34}$ causes atoms on the surface of the nanoparticles to accumulate in a 


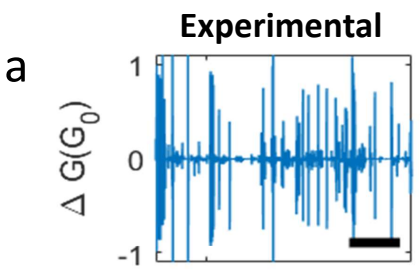

$\mathrm{t}(\mathrm{s})$

b

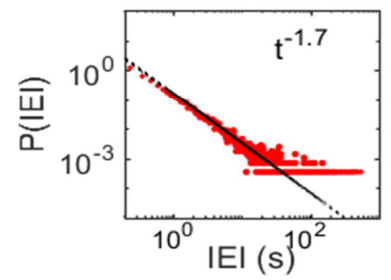

C

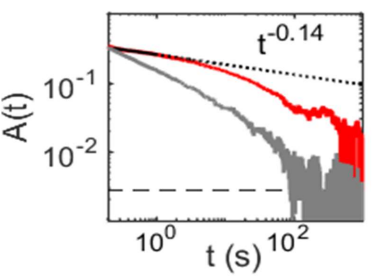

d

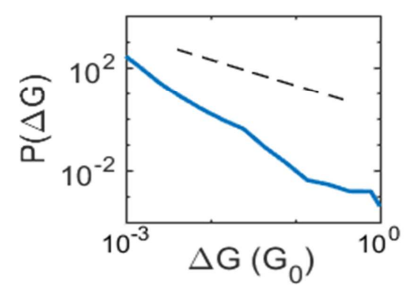

Probabilistic

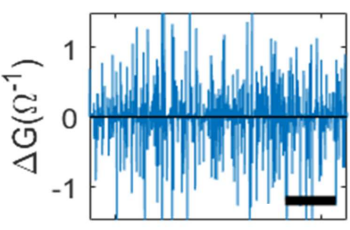

$\mathrm{t}(\mathrm{s})$
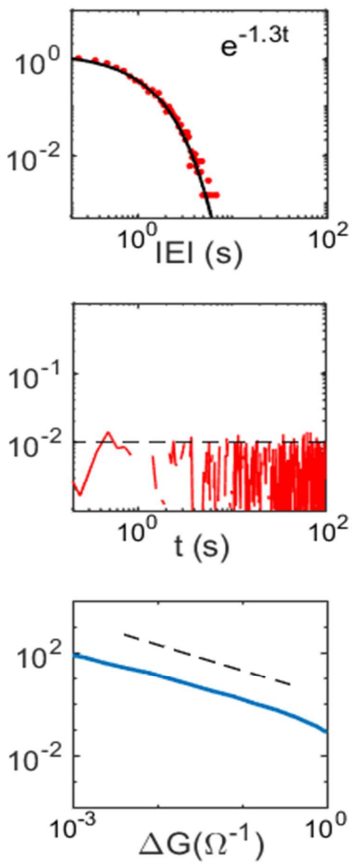

Deterministic

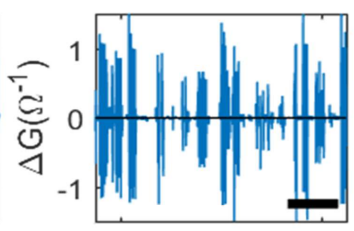

$\mathrm{t}(\mathrm{s})$
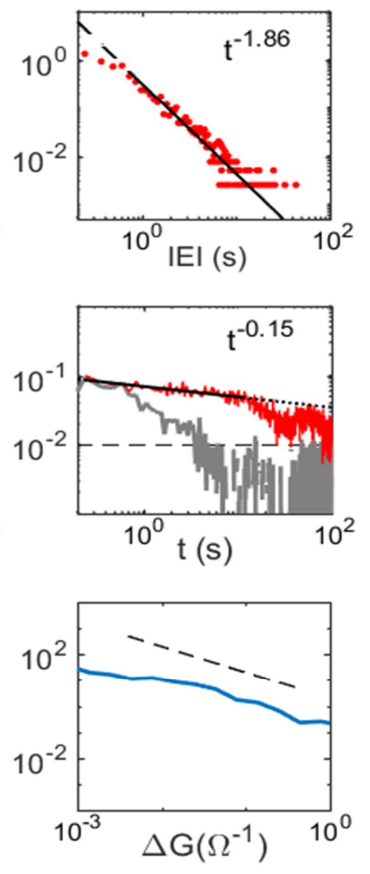

Figure 2: Switching dynamics in the experiments (left column), probabilistic-model (centre column) and deterministic model (right column). (a) Experimentally observed bursty behavior, is reproduced by the deterministic model but is absent in the probabilistic model (scale bar $=2000 s)$. (b) The distribution of inter-event intervals $(\mathrm{P}(\mathrm{IEI})$ ) is a power law for the experimental data and the deterministic model (with a similar slope) but decays exponentially for the probabilistic model (black: maximum likelihood fit). (c) Autocorrelation function $A(t)$ (red) is a power law for both experimental data and deterministic model with $t^{-0.14}$, but is essentially zero (i.e. below the confidence bound (dashed lines)) for the probabilistic model. Shuffling the IEI sequence (grey) leads to a lower $A(t)$ (see Ref. 29,30 and refs therein). (d) Distribution of event sizes $(\mathrm{P}(\Delta G)$, blue) exhibits a heavy-tail in all cases. The black dashed line (slope $=-1$ ) is a guide to the eye. The slopes in the simulations are smaller (by a factor of 2) than observed experimentally due to the smaller system size in the simulations.

'hillock' (yellow in Figure 1e), decreasing the size of the tunnel gap. During the second pulse, filament formation nearly reaches completion but because the applied electric field is close to the threshold for inducing atomic motion, fluctuations in the conductance are observed. 
Towards the end of the pulse, the 'hillock' of atoms relaxes so that the conductance returns to a value near to its initial level. During the third pulse, the hillock of atoms extends completely across the tunnel gap, forming a filament (red in Figure 1e) that has relatively high conductance (of the order of the quantum of conductance, $G_{0}=2 e^{2} / h$ ). ${ }^{25}$ This switching event causes an increase in observed network conductance, as well as a reduction of the potential difference between the two groups of nanoparticles. Figure 1d shows the reverse process, i.e., over three voltage pulses electromigration effects ${ }^{35}$ reduce the average width of a previously formed filament until it is broken, causing a clear decrease in the network conductance.

An important feature of these results is that the effect of the applied electric field/current is cumulative, i.e., both formation and destruction of the atomic scale filaments can be viewed as integrating the applied signals until filament formation/destruction ('firing'). The fluctuations ${ }^{40}$ in Figure 1c are consistent with surface energy effects, ${ }^{41}$ which attempt to return the gap/filament to its original size ('leak') when there is no current/voltage. As is shown in more detail in Figure S1 these processes are therefore qualitatively similar to leaky integration and fire (LIF) mechanisms in biological neurons. ${ }^{42,43}$

We now show, using computer simulations, that when coupled with the intrinsically scalefree architecture of the percolating-tunneling network, ${ }^{30}$ this local integrate and fire (IF) mechanism leads to long-range temporal correlations and the generation of the avalanches of events that are very similar to those observed experimentally. [Note that detailed modelling of the atomic scale processes that lead to the LIF dynamics is potentially very interesting but would require significant extensions of the models of Refs. 33,34,38.] The experimental results are summarised in the left columns of Figures 2 and 3 - see captions and Methods for details. The essential points are (i) distributions of inter-event intervals (IEIs) and the autocorrelation functions (ACFs) in Figure 2 are power laws, which are characteristic of long-range temporal correlations (LRTC) ${ }^{30}$ and (ii) the power law avalanche distributions in Figure 3 are consistent with criticality. ${ }^{29,44}$ The heavy-tailed $\Delta G$ distributions (Figure $2 \mathrm{~d}$ ) 
reflect the dynamical nature of the network, as discussed below.

Numerical simulations have been used previously to show that the experimental networks of nanoparticles are well described by continuum models ${ }^{37,45}$ in which the conducting objects are represented by uniform discs, which are allowed to overlap, representing formation of groups of particles. ${ }^{37,46}$ Below the percolation threshold $\left(p<p_{c}\right)$, no single group spans the entire network and the conduction of the system is due to the tunnel currents flowing across small tunnel gaps which separate the groups of particles. It is assumed that the groups are large enough that both the charging energy of a connected group and the quantization of energy levels are negligible, and that the resistance between overlapping particles within a group is negligible, so that the only resistance in the system is due to the tunnel gaps. Each gap is assigned a conductance, $G_{i}=A \exp \left(-\delta L_{i}\right)$, where $A$ and $\delta$ are constants and $L_{i}$ is the size of the gap (in units of the particle diameter which is set to $1 ; A=1 \Omega^{-1}$ and $\delta=100$ for convenience). ${ }^{37}$ After the formation of a filament, the gap is assigned a conductance $G=10 \Omega^{-1}$; the precise conductance values are not important and could be scaled to match the experiments more closely, but we choose to maintain consistency with previous work. ${ }^{37}$ We focus primarily on simulations of systems with a size of $L \times L$ particle diameters $(L=200$ is chosen to provide the best trade-off between computational time and finite-size effects) and surface coverage $p<p_{c}$, but the results are substantially the same for $0.64 \leq p<p_{c}$ and for $200 \leq L \leq 400$, consistent with Ref. 30 .

We first consider a probabilistic model. ${ }^{46}$ When the electric field in a gap or the current in a filament is greater than a threshold value, the switch is allowed to change state (switch on $(\uparrow)$ or off $(\downarrow)$ ) with a well-defined probability (here $p_{\uparrow}=p_{\downarrow}=0.001$, but the results are qualitatively independent of the parameter values). This model allows demonstration of interesting switching behaviour (and in particular the formation of connected pathways across the network ${ }^{46}$ ), as well as consequent redistribution of voltages and currents through the network. However, the results in the centre column in Figure 2(b, c) show that the IEI distribution is exponential (not power law, as in the experiments), and the corresponding 

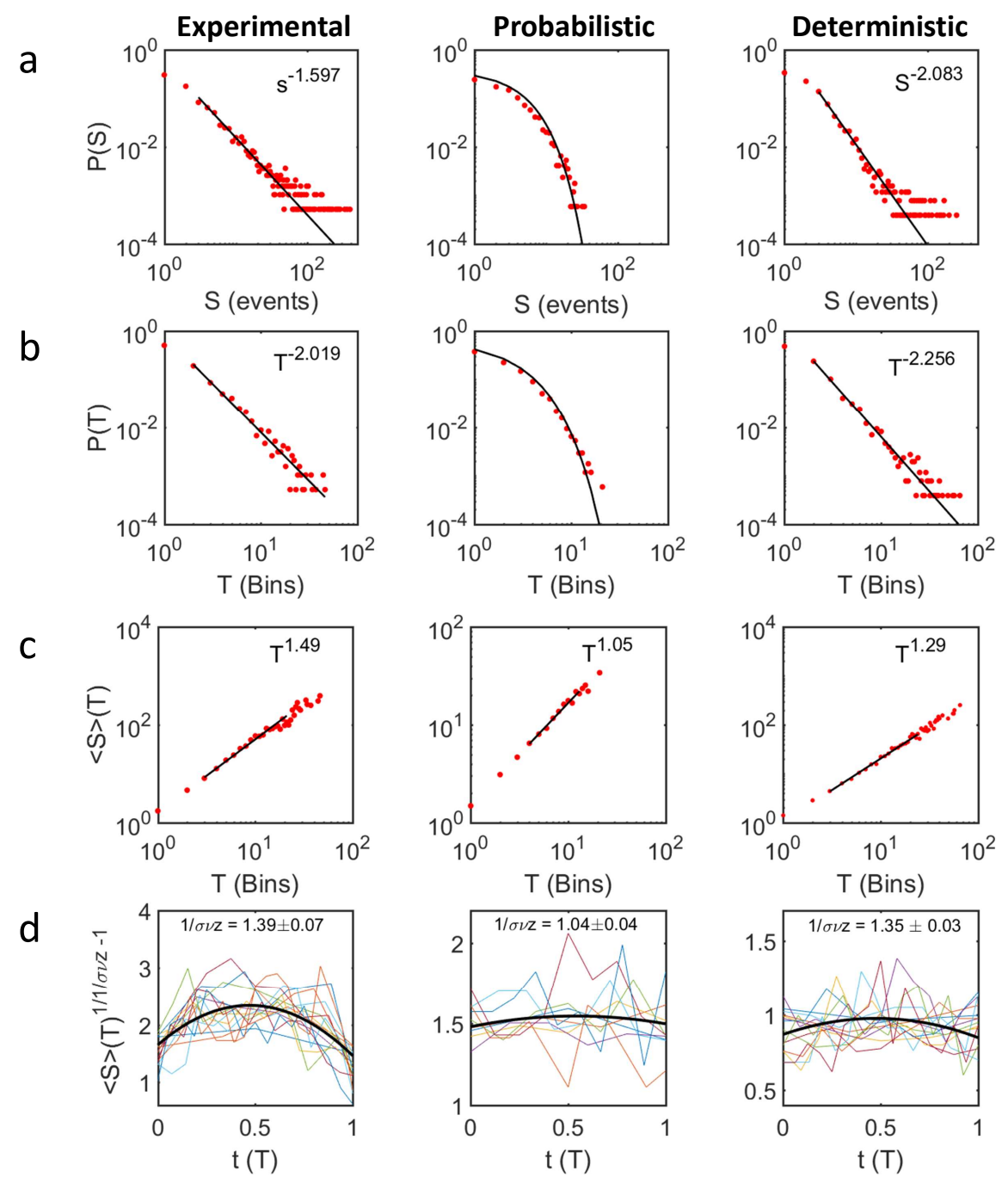

Figure 3: Avalanche and criticality analysis for the experimental data (left column), probabilistic model (centre column) and deterministic model (right column). (a and b) Sizes $(S)$ and durations $(T)$ of the avalanches are distributed as power laws for the experimental data and the deterministic model, with slopes that are the same to within $\lesssim 20 \%$, but the probabilistic model results in exponential distributions (black: maximum likelihood fit). (c) Average avalanche size for given duration $\langle S\rangle(T) \sim T^{1 / \sigma \nu z}$ with exponent $1 / \sigma \nu z \sim$ 1.3-1.5 for experimental data and deterministic model (a difference of only $\sim 10 \%$ ), whereas probabilistic model yields $1 / \sigma \nu z \sim 1$. (d) Average avalanche shapes for each duration showing collapse onto a universal scaling function (black line), and yield independent measures of the critical exponent $1 / \sigma \nu z$. The power law behaviour and agreement of estimates of $1 / \sigma \nu z$ for the experimental data and deterministic model are consistent with criticality. ${ }^{29,44}$ See also Table 1.

ACF shows an absence of correlations. Similarly, the centre column of Figure 3(a, b) shows that the distributions of avalanche sizes $(S)$ and durations $(T)$ are exponential. The absence 
of correlations is not surprising - in the probabilistic model the switching events occur randomly, and so there is no possibility that correlated avalanches can emerge.

We now consider a new deterministic model which captures the atomic scale dynamics of the switching process described in Figure 1. To emulate the experimentally observed behaviours, the size of each tunnel gap $\left(d_{i}\right)$ changes in response to the electric field $E_{i}$ in the gap according to

$$
\Delta d_{i}= \begin{cases}r_{d}\left(E_{i}-E_{T}\right), & \text { if } E_{i} \geq E_{T} \\ 0, & \text { otherwise }\end{cases}
$$

and the current flow $\left(I_{j}\right)$ in each existing filament causes electromigration effects ${ }^{35}$ that decrease its width $\left(w_{j}\right)$ according to

$$
\Delta w_{j}= \begin{cases}r_{w}\left(I_{j}-I_{T}\right), & \text { if } I_{j} \geq I_{T} \\ 0, & \text { otherwise }\end{cases}
$$

where $r_{d}$ and $r_{w}$ are parameters that control the rates at which $d$ and $w$ change when threshold fields $\left(E_{T}\right)$ and currents $\left(I_{T}\right)$, respectively, are exceeded. Here $E_{T}=10 \mathrm{~V}$ and $I_{T}=0.01 \mathrm{~A}$, which are chosen to be consistent with estimates obtained from experiments. ${ }^{25}$ The right column in Figure 2(b, c) shows that the deterministic model reproduces the power law IEI distribution and strong correlations observed in the experiments. The right column in Figure 3(a, b) shows that the deterministic model also reproduces the power law distributions of $S$ and $T$. Furthermore, for the deterministic simulations, the three different estimates of the critical parameter $1 / \sigma \nu z$ shown in Table 1 are in good agreement, therefore satisfying rigorous criteria for criticality. ${ }^{29,31,44}$ The deterministic simulations are in excellent qualitative agreement with the experiments for a broad range of parameters (see below), while the probabilistic simulations fail to reproduce the observed power law behaviour. The already good quantitative agreement between the deterministic simulations and the experiments in Figures 2 and 3 could most likely be improved even further by 
Table 1: Criticality in the experiments and deterministic simulations. The critical exponent $1 / \sigma \nu z$ is obtained from the crackling relationship $(\alpha-1) /(\tau-1)$, mean avalanche size given duration $\langle S\rangle(T)$, and avalanche shape collapse for both representative experimental data and the deterministic simulations. The agreement of these three independent estimates of $1 / \sigma \nu z$ is a rigorous requirement for criticality. See Refs. $29,31,44$ for details of the criticality analysis.

\begin{tabular}{|l|l|l|l|l|l|}
\hline Exponents & $\tau$ & $\alpha$ & $\begin{array}{l}\text { Crackling } \\
\text { relationship }\end{array}$ & $<S>(T)$ & $\begin{array}{l}\text { Shape } \\
\text { collapse }\end{array}$ \\
\hline Exp. & $1.6 \pm 0.1$ & $2.0 \pm 0.1$ & $1.7 \pm 0.2$ & $1.49 \pm 0.03$ & $1.39 \pm 0.07$ \\
\hline Det. Sim. & $2.1 \pm 0.1$ & $2.6 \pm 0.1$ & $1.2 \pm 0.2$ & $1.29 \pm 0.02$ & $1.35 \pm 0.03$ \\
\hline
\end{tabular}

fine-tuning the model parameters. The optimum simulation parameters are expected to be material dependent.

The deterministic simulations allow the generation of maps of the current and voltage distributions in the network at each time step, providing a method to elucidate the mechanism for the propagation of the critical avalanches (Figure S2 and video V1). When an external stimulus (voltage) is applied, the formation (or annihilation) of an atomic filament at a tunnel gap redistributes current across the entire network, thereby modifying local electric fields in other tunnel gaps. This in turn changes the rates at which tunnel gaps/filaments change size (see Eqs. 1 and 2), leading to further switching events. In other words, each switching event influences subsequent switching events through internal feedforward and feedback, giving rise to temporal correlations. We emphasise that it is this correlated switching behaviour in the deterministic simulations that leads to the critical dynamics; the absence of the correlated switching in the probabilistic case leads to non-critical dynamics.

We now turn to a discussion of the distribution of $\Delta G$ values which, as shown in Figure 2d, are heavy-tailed for both experimental and simulational data. Both the deterministic and probabilistic models generate similar distributions (Figure $2 \mathrm{~d}$ centre and right panels). The $\Delta G$ distributions for the ensemble of switches, reflect both the positions of the individual switches in the network, and the number of times the switches open or close. ${ }^{30}$ Figure S3 reveals that the values of $\Delta G$ measured for individual switching sites are also distributed over several orders of magnitude. As shown in Figure S4, this surprising result is due to 

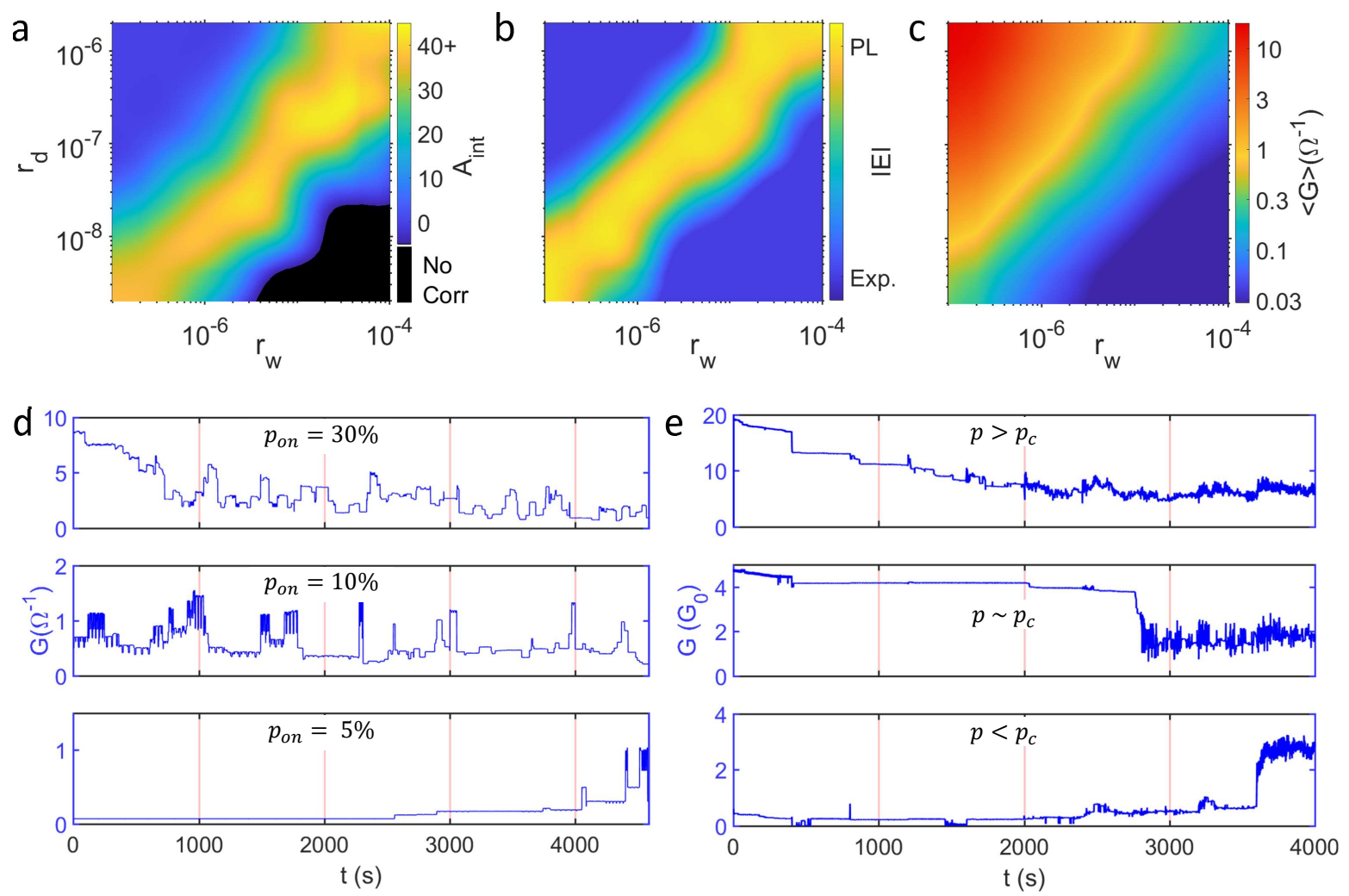

Figure 4: Maps of parameter space $\left(r_{d}, r_{w}\right.$ - see Eqs. 1,2) for the deterministic model showing a self-tuned critical state. (a) Strength of correlations (integrated autocorrelation $A_{\text {int }}$ - see Methods) and (b) Characterisation of the power law (PL) and exponential (Exp.) fits to the IEI distribution. Both (a) and (b) show a clear ridge $\left(r_{d} \sim r_{w} / 25\right)$ corresponding to strong correlations and criticality. (c) Average network conductance showing that the ridge in parameter space corresponds to $\langle G\rangle \sim 0.5 \Omega^{-1}$. Note that the parameters chosen to illustrate the deterministic simulations in Figures 2 and 3 correspond to a point on this ridge $\left(r_{d}=5 \times 10^{-7} V^{-1}, r_{w}=3 \times 10^{-5} A^{-1}\right)$. (d) Initial states in which $5 \%, 10 \%$ and $30 \%$ of switches are 'on' all self-tune towards $G \sim 0.5 \Omega^{-1}$. (e) Corresponding experimental data showing that under voltage stimulus devices with different surface coverages self-tune towards critical states with $1 \lesssim G \lesssim 6 G_{0}$. The difference between optimum values of $G$ in the experiment and simulation results from the choice of simulation parameters, which maintains consistency with previous work. ${ }^{37}$

dynamical reconfiguration of the network: at different times each switch can find itself on different branches of the (fractal) ${ }^{30}$ arrangement of current paths. Hence, even though the change in conductance of any individual switch is essentially the same, each time the switch changes state the configuration of the rest of the network is different so a different value of $\Delta G$ is measured. 
Finally, we discuss the range of parameter space in the deterministic model in which correlations and critical avalanches are observed. Figures 4a and 4b show that strong correlations and power law IEI distributions are obtained along a diagonal ridge in the $r_{d}, r_{w}$ parameter space. Figure $4 \mathrm{c}$ shows that this ridge corresponds to a narrow range of conductances, where the connectivity of the network is optimised for criticality: the number of switches in their 'off' or 'on' states is 'balanced'. The nature of the balanced state is illustrated by the results of simulations in which the initial state of the network is chosen to have different numbers of switches in the 'on' state (Figure 4d). When the number of switches that are 'on' is high, the resulting high current will tend to break filaments and return the system to the balanced state. Conversely, if the number of switches that are 'on' is low, higher electric fields in some tunnel gaps will cause additional switches to turn 'on', again returning the system to the balanced state. Hence the system always self-tunes to a dynamical state where the number of pathways through the network is close to an optimum value. This 'balance' is essential for critical avalanches to propagate. ${ }^{15,39}$

Figure $4 \mathrm{e}$ shows that the experimental system self-tunes to achieve a similar balance. If the initial conductance of the network ( $G_{\text {init }}$, measured immediately after deposition) is either higher or lower than the narrow range $\left(1 \lesssim G \lesssim 6 G_{0}\right)$ in which correlations and criticality are observed, ${ }^{29}$ switches change state so as to move the system back into that $G$ range.

In summary, we have presented experimental evidence for atomic-scale integrate and fire mechanisms within our percolating networks and shown by detailed modelling that these processes facilitate critical avalanches. Both experimental and simulational results are consistent with optimally balanced network states similar to the self-organized-critical states reported in biological neuronal networks. ${ }^{47,48}$ These results provide a significant step towards understanding the dynamics of nanoscale switching networks, and will facilitate the development of applications. For example, as discussed in some detail in Refs. 29,30, performance of pattern recognition algorithms based on reservoir computing ${ }^{5,17,18}$ is believed to 
be optimised for scale-free ${ }^{16}$ and critical ${ }^{14,15}$ networks, and we believe there are many new opportunities to be explored in the field of unsupervised learning. ${ }^{10}$

\section{Methods}

Experimental methods and analysis have been described in detail in Refs. 29 and 30, and so we provide here only a brief summary.

Device fabrication. Our percolating devices are fabricated by simple nanoparticle deposition processes. ${ }^{25,26,49} 7 \mathrm{~nm}$ Sn nanoparticles are deposited between gold electrodes (spacing $100 \mu \mathrm{m})$ on a silicon nitride surface and coalesce to form particles of $20 \mathrm{~nm}$ diameter. Deposition is terminated at the onset of conduction, which corresponds to the percolation threshold. ${ }^{36,49}$ The deposition takes place in a controlled environment with a well-defined partial pressure of air and humidity, as described in Ref. 26. This process leads to controlled coalescence and fabrication of robust structures which function for many months, but which yet allow atomic scale switching processes to take place unhindered.

Electrical stimulus and measurement. Electrical stimuli are applied to the electrode on one side of the percolating device, while the opposite electrode of the system is held at ground potential. DC Measurements over long time periods are necessary to avoid significant cut-offs in power law distributions. ${ }^{50,51}$ Pulsed measurements are used to probe atomic scale dynamics. The conductance measurements reported here are performed with $100 \mathrm{~ms}$ sampling intervals, but we have shown previously ${ }^{29,30}$ that quantitatively the same behavior is observed for much shorter sampling intervals.

Data Analysis. The data analysis methods used to identify avalanches of switching events are substantially the same as those developed in the neuroscience community to analyse micro-electrode array recordings from biological brain tissue, and described in detail in Ref. 29 .

To quantify the correlations in the simulated event trains, we use the Autocorrelation 
Function (ACF). Since the initial values of the ACFs (commonly called 'lag-1') may be affected by finite sampling rate, we use the integrated value of the $\mathrm{ACF}$ from $t=0$ to $1000 \mathrm{~s}$ as an indicator of the correlation strength, and we use the slope of the ACF to quantify the timescale of the correlations.

Following Refs. 29,31, both in the experiments and simulations, the size $(S)$ and duration $(T)$ of each avalanche of signals is defined by counting the total number of events in the avalanche and the number of time bins over which the avalanche propagates. The time bins have widths corresponding to the mean IEI.

Fitting and goodness-of-fit. As described in detail in Ref. 29 we follow the maximum likelihood (ML) approach of Ref. 50,51 to estimate power law exponents in the IEI and avalanche size distributions. The ML estimators are obtained for both power law and exponential distributions. We use the Akaike information criterion ${ }^{52}$ to identify which distribution is more likely and find in all cases that it is the power law. In all cases, we fail to reject the null hypothesis that distributions are power-law-distributed (we require $p$-values $>0.2$ ), but we do reject the null hypothesis that the distributions are exponentially distributed (we find $p$-values $<0.01)$. We do not fit the event size $(\Delta G)$ distributions because the precise shape of the distributions is not important to the analysis - they are however, well-fitted by long-tailed functions such as a weakly truncated power law.

ML methods cannot be applied to data which is not in the form of a probability distribution and so the standard linear regression techniques are used to obtain the measured exponents for $A(t)$ and $<S>(T)$.

\section{Supporting Information}

The Supporting Information is available free of charge on the ACS Publications website at DOI: *******.

(1) Leaky integrate and fire (LIF) mechanism due to atomic-scale dynamics; (2) Simulations showing mechanism for propagation of critical avalanches; (3) Large variation of measured 
values of change in network conductance $(\Delta G)$; (4) Dynamical reconfiguration of the network; (5) Effect of additional conduction pathways on the $\Delta G$ distribution; (6) Comparison between percolating and memristive devices such as physically evolving networks; (pdf) Avalanche propagation in the simulated network (MP4)

\section{AUTHOR INFORMATION}

\section{Corresponding Author}

E-mail: simon.brown@canterbury.ac.nz

\section{ORCID}

Matthew D. Pike: 0000-0003-0178-7629

Saurabh K. Bose: 0000-0001-8848-5097

Joshua B. Mallinson: 0000-0001-6611-245X

Susant K. Acharya: 0000-0002-1912-2521

Shota Shirai: 0000-0003-4125-6195

Edoardo Galli: 0000-0002-2356-2837

Stephen J. Weddell:

Philip J. Bones:

Matthew D. Arnold: 0000-0003-4164-0242

Simon A. Brown: 0000-0002-6041-4331

\section{Author Contributions}

S.A.B conceived the study and initiated the project. M.D.P and S.K.B contributed equally to this work. S.K.B, J.B.M, S.K.A, E.G, and S.S performed the experiments and associated data analysis. P.J.B. and M.D.P. proposed the deterministic simulations. M.D.P and M.D.A performed the numerical simulations. S.J.W and P.J.B. helped with the numerical simulations. S.A.B., S.K.B, and M.D.P wrote the manuscript with comments from all 
authors.

285

286

${ }_{287}$ Acknowledgement

\section{Notes}

This project was financially supported by The MacDiarmid Institute for Advanced Materials

289

290

The authors declare that they have no competing financial interests. and Nanotechnology, the Ministry of Business Innovation and Employment, and the Marsden Fund. 
291 Supplementary Figures 
a

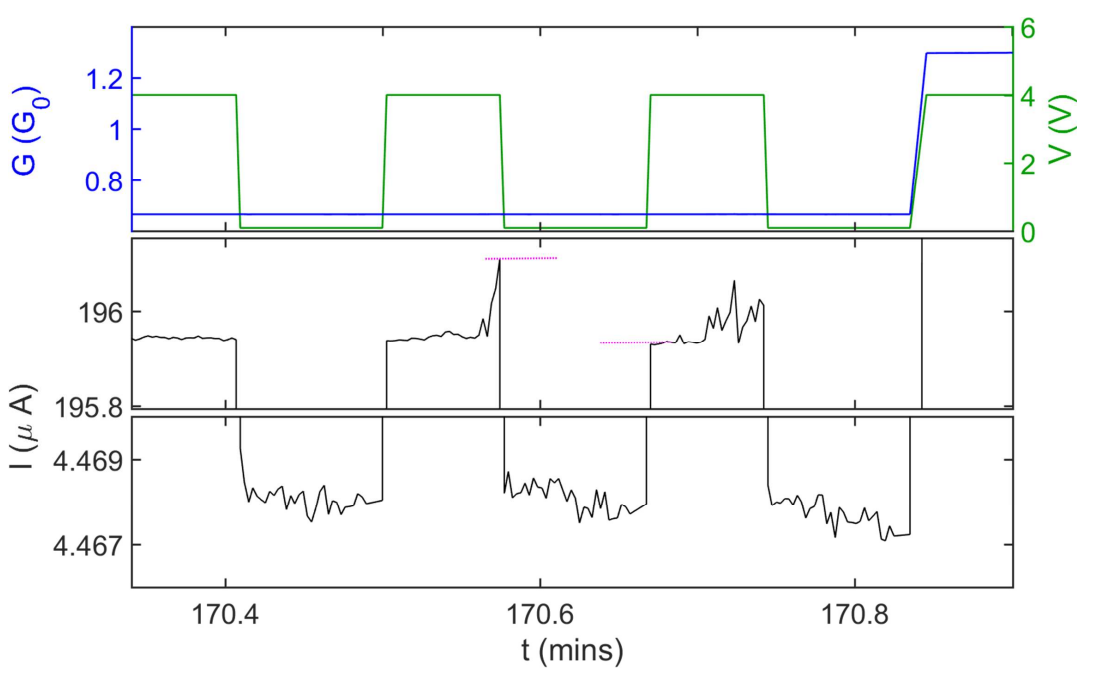

b

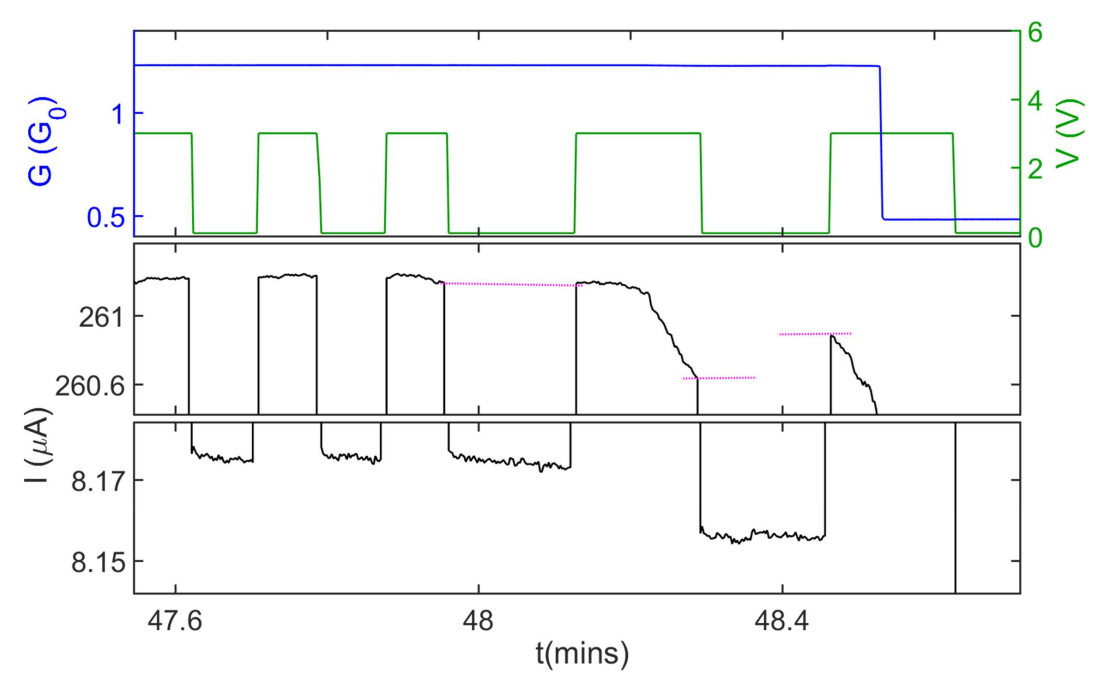

Figure S1: Leaky integrate and fire (LIF) mechanism due to atomic-scale dynamics. (a) Applied voltage pulses (green) cause the formation of an atomic-scale hillock in one tunnel gap (see schematic in Figure 1e). The hillock reduces the size of the tunnel gap and slightly increases the current (black). The corresponding increase in conductance (blue) is imperceptible on this scale. When the applied voltage returns to a 'read' level $(0.1 \mathrm{~V}$ between pulses) before the atomic-filament formation process is complete, the hillock relaxes back ('leaks'), recovering the original current level (as emphasised by pink lines). After several pulses, the hillock grows across the tunnel gap (Figure 1e) and atomic-filament formation is completed, resulting in a switching event with a large change in conductance ('fire'). (b) Similarly, when filaments start to break due to electromigration it is possible to see a partial recovery of the current during the time between voltage pulses. The pink lines again show that this is a 'leaky' process. Again, signal integration resumes during the subsequent pulse and is completed when the filament breaks completely (large decrease in $G$ ). 


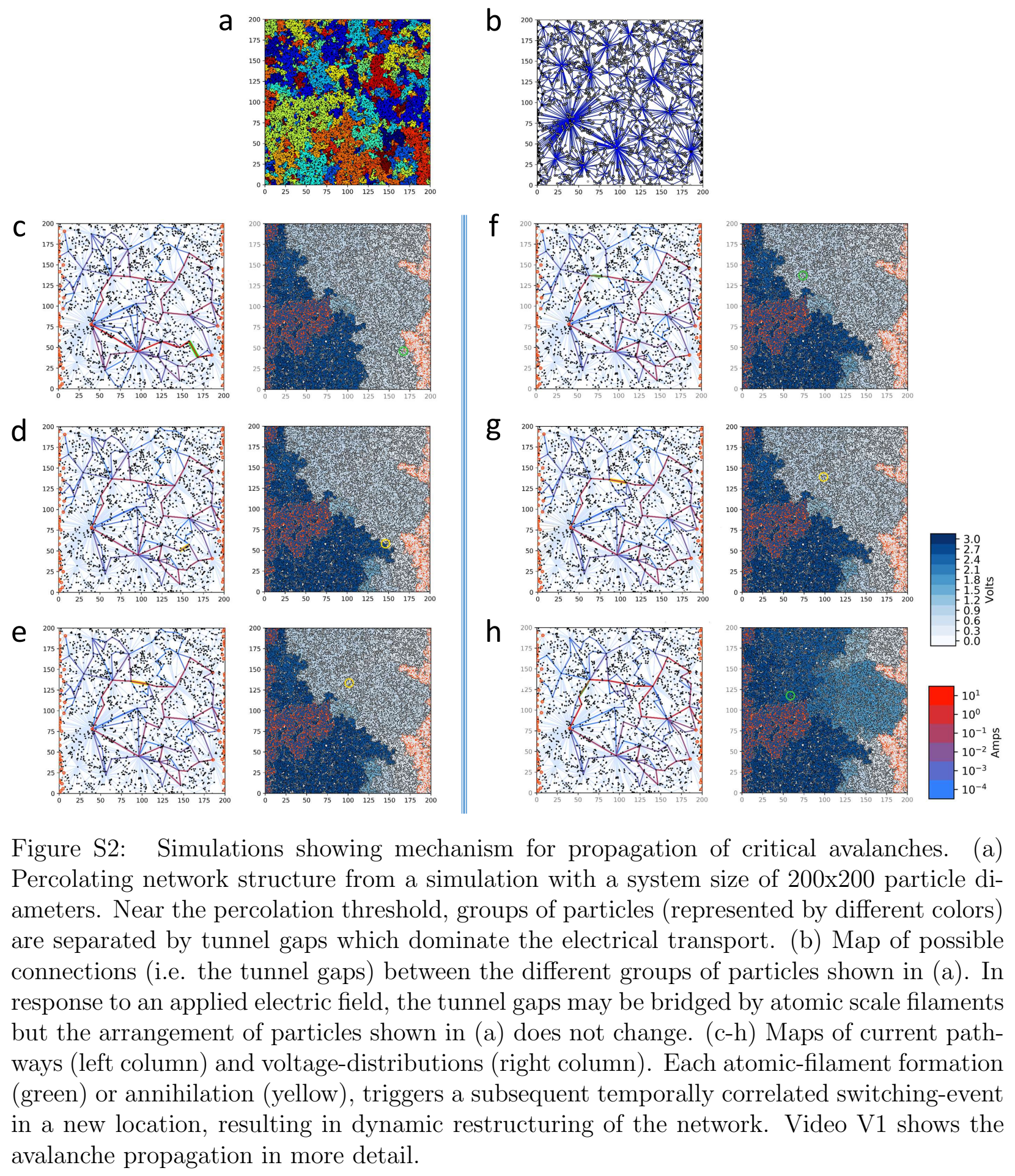

Figure S2: Simulations showing mechanism for propagation of critical avalanches. (a) Percolating network structure from a simulation with a system size of 200x200 particle diameters. Near the percolation threshold, groups of particles (represented by different colors) are separated by tunnel gaps which dominate the electrical transport. (b) Map of possible connections (i.e. the tunnel gaps) between the different groups of particles shown in (a). In response to an applied electric field, the tunnel gaps may be bridged by atomic scale filaments but the arrangement of particles shown in (a) does not change. (c-h) Maps of current pathways (left column) and voltage-distributions (right column). Each atomic-filament formation (green) or annihilation (yellow), triggers a subsequent temporally correlated switching-event avalanche propagation in more detail. 


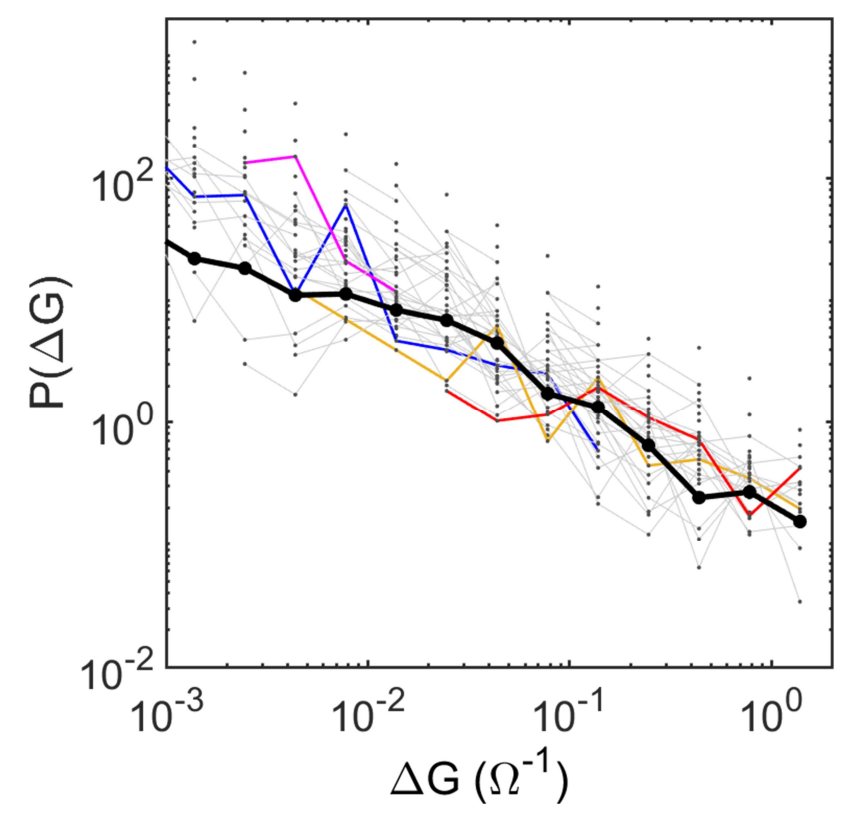

Figure S3: Large variation of measured values of change in network conductance $(\Delta G)$. The distribution of event sizes $\mathrm{P}(\Delta G)$ in the deterministic simulations is shown, both for a large number of individual sites within the network (grey) and for all sites (black). The data from each of four representative sites are highlighted with different colours showing that events occuring at the same site can lead to very different $\Delta G$. The broad range of $\Delta G$ values is generated because the complex network is constantly being restructured and so each time an event occurs at one physical location the network around it is different - see Figure S4. 
a

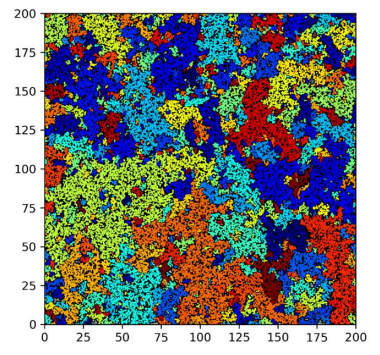

C

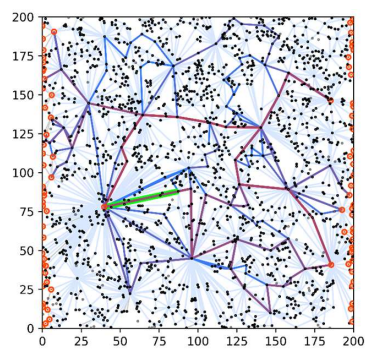

d

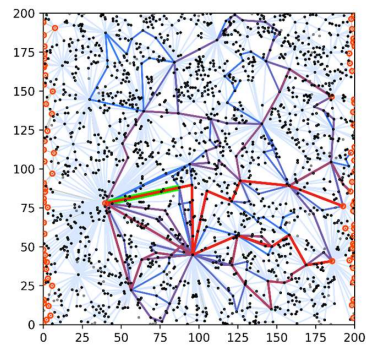

e

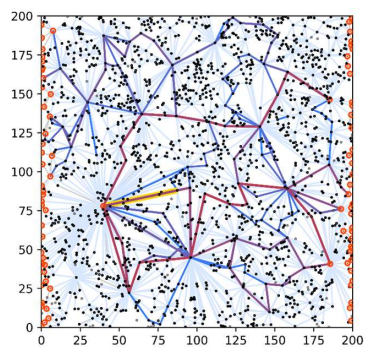

f

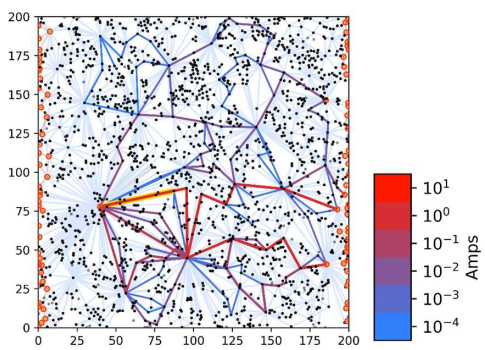

b
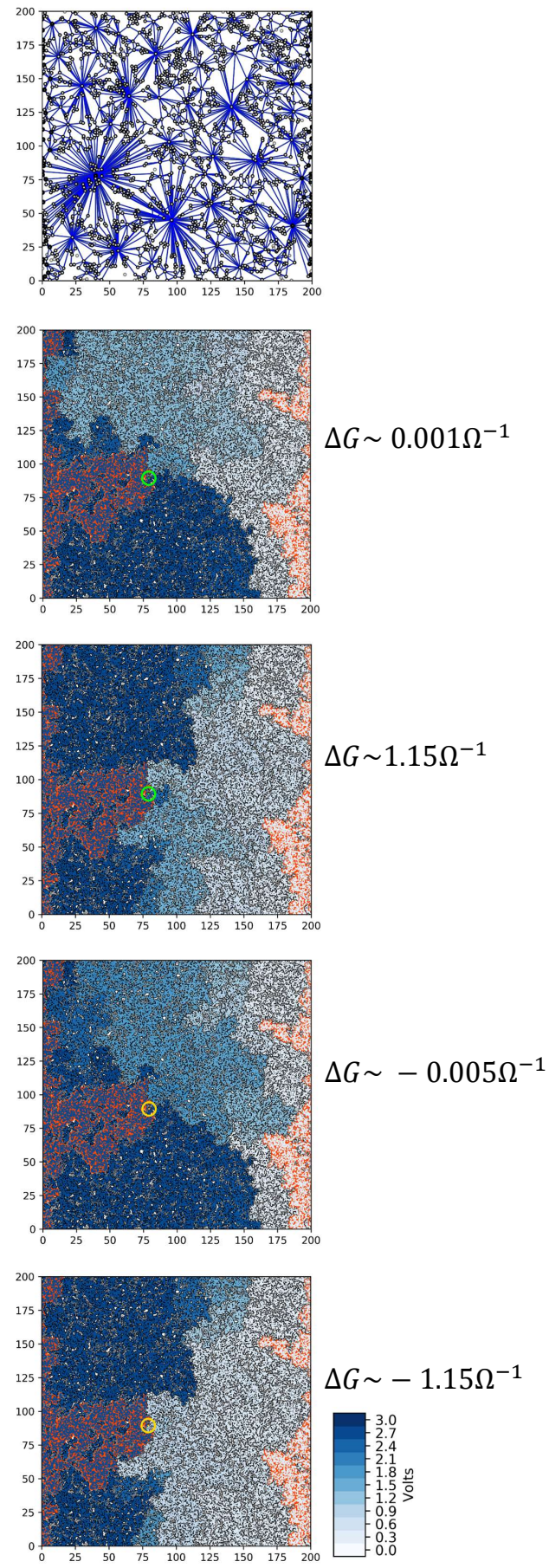

Figure S4: Dynamical reconfiguration of the network. (a) Percolating network structure and (b) map of possible connections (i.e. tunnel gaps) for the same parameters as in Figure S2. (c-f) The plots show examples of the distribution of current pathways (left column) and voltages (right column), after switching at a single site (highlighted with green and yellow, corresponding to increases and decreases in $\Delta G$ respectively). Due to reconfiguration of the network, in these examples switching at this one site results in the network conductance $G$ undergoing (a) a small increase $\sim 0.001 \Omega^{-1}$, (b) a large increase $\sim 1.15 \Omega^{-1}$, (c) a small decrease $\sim-0.005 \Omega^{-1}$ or $(\mathrm{d})$ a large decrease $\sim-1.15 \Omega^{-1}$. 

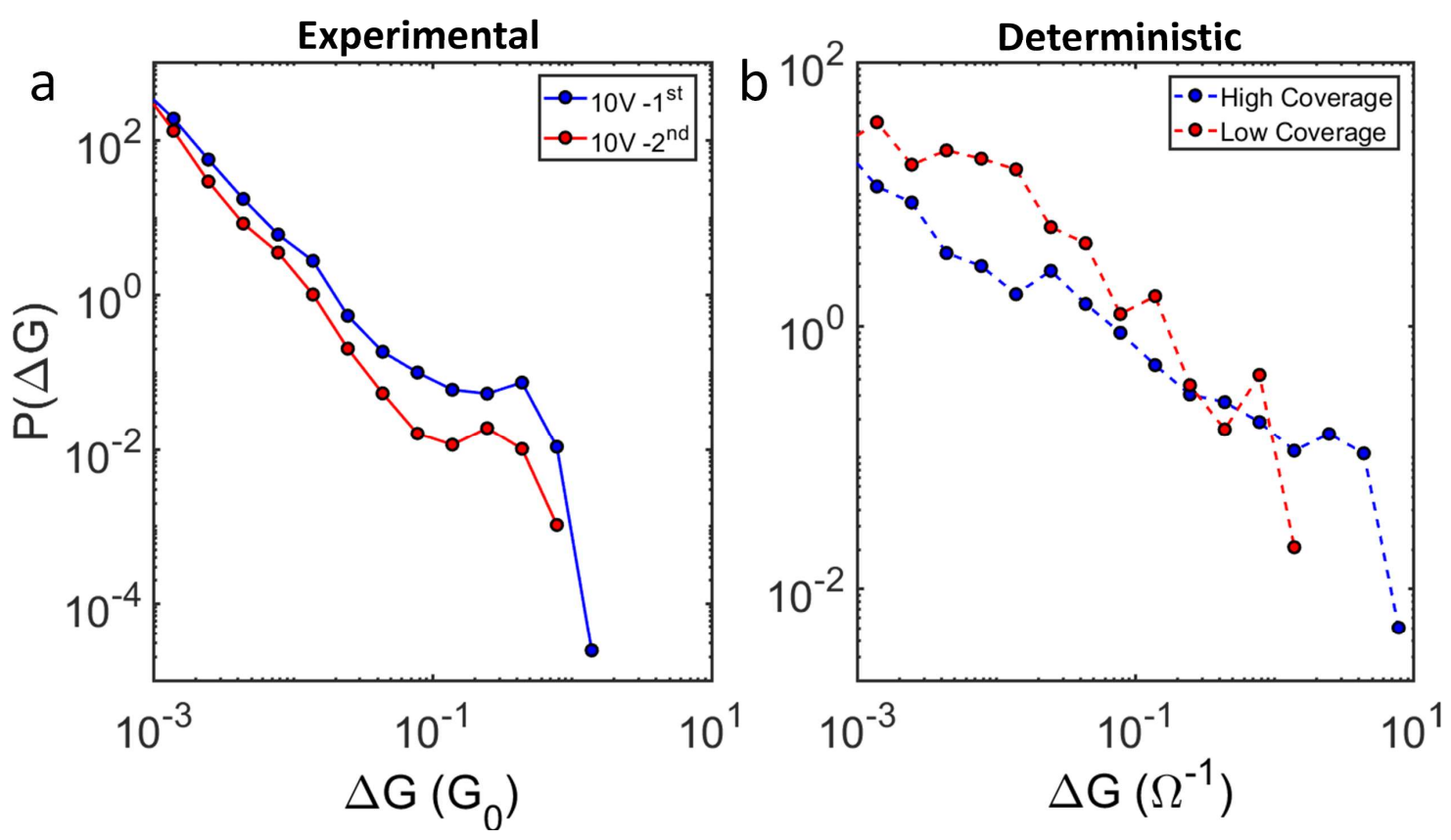

Figure S5: Effect of additional conduction pathways on the $\Delta G$ distribution. (a) Experiment. Application of high voltages (first measurement; blue) results in destruction of filaments connecting groups of particles so that during the second measurement (red) the slope of $\mathrm{P}(\Delta G)$ decreases and there is an absence of events with large $\Delta G$. (b) Similar effect in the deterministic model. At low surface coverage (red) there are less conducting pathways and therefore a lower slope and absence of large $\Delta G$ events. 
Comparison between percolating and memristive devices such as physically evolving networks

The differences in the structure of our percolating networks of nanoparticles ${ }^{25,29,37,46,49}$ and the arrangement of nanoparticles in certain memristive devices ${ }^{4,9,38}$ is highlighted in Figure S6. For simplicity we focus on a comparison with the physically evolving networks (PENs) described in Ref. 38. The essential point is that in Ref. 38 the structure of the nanoparticle assembly changes after signals are applied: physical tracks through the particle network are observed after signals are applied. In contrast, in our devices no changes to the arrangement of nanoparticles occur at the usual operating voltages.

For completeness we make the following further detailed comments on the distinctions between our devices and those of Ref. 38 .

1) We present SEM images showing the lack of structural changes in our devices at moderate voltages in Figure S7. We include also images of devices exposed to higher voltages which do show physical restructuring. We emphasise such restructuring is observed only at high voltages.

2) While it is common in the literature to describe devices such as PENs ${ }^{38}$ as 'percolating', percolation theory ${ }^{36}$ requires that the components (the nanoparticles) fill space randomly, which is indeed the case in our devices (see Refs. 25-27,30 and especially Refs. 29,36,37,46,49 for a detailed description) but is not the case in Ref. 38 .

3) In Ref. 38 pathways through the network are formed by 3 processes (ionisation, migration and reduction) that lead to restructuring of the nanoparticles and formation of nanoscale filaments that connect the electrodes. Similar processes are at work in our devices but additionally field-induced diffusion and evaporation are important. ${ }^{33,34,38}$ These processes take place on a local scale (i.e. between the nanoparticles) and hence lead to atomic scale switching processes. ${ }^{11,12,22}$

4) Typical memristive devices ${ }^{4,9,38}$ have sub-micron separations between electrodes, whereas our devices are $100 \mu \mathrm{m}$ across. Since the applied voltages are the same in Ref. 38 as in our 
work, in our case the larger network means that active electric field near each particle is much smaller and wholesale re-arrangements of the nanoparticles are not possible (see Figure S7). Furthermore, the small devices of Ref. 38 do not allow the complexity and long-range spatial correlations required for criticality.

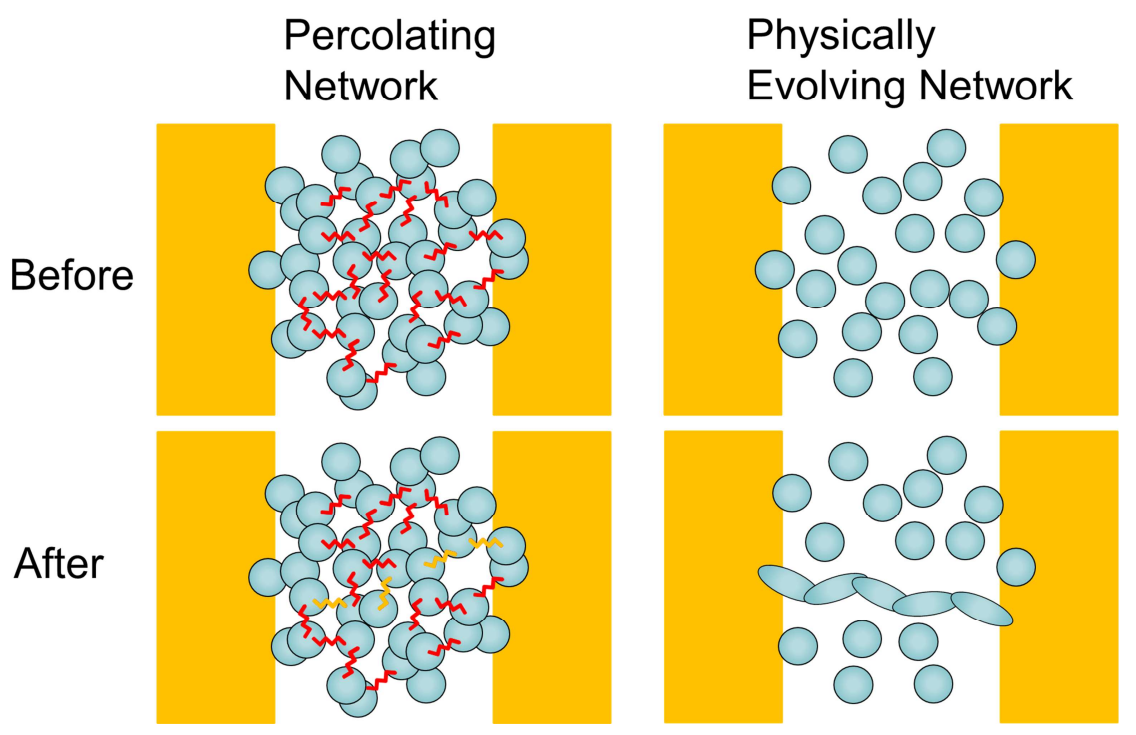

Figure S6: Illustration of the structure of the percolating network and comparison with memristive devices which exhibit formation of nanoscale conduction paths, as exemplified by the physically evolving networks (PENs) described in Ref. 38. Top row: before application of input signals (voltages). The percolating network consists of groups of particles that can be modelled as overlapping discs ${ }^{37}$ whereas the PEN comprises discrete particles formed by diffusion and aggregation processes. ${ }^{4,9,38}$ Bottom row: after application of input signals (voltages). The physical structure of the percolating network is unchanged, but some tunnel gaps (red symbols) are bridged by atomic-scale filaments (orange symbols) that connect groups of particles. Hence the conductivity in the network is modified by the formation (and subsequent breaking) of atomic filaments. In the PEN the application of voltages causes modifications of the physical structure and the formation of nanoscale conducting filaments which connect the electrodes. 


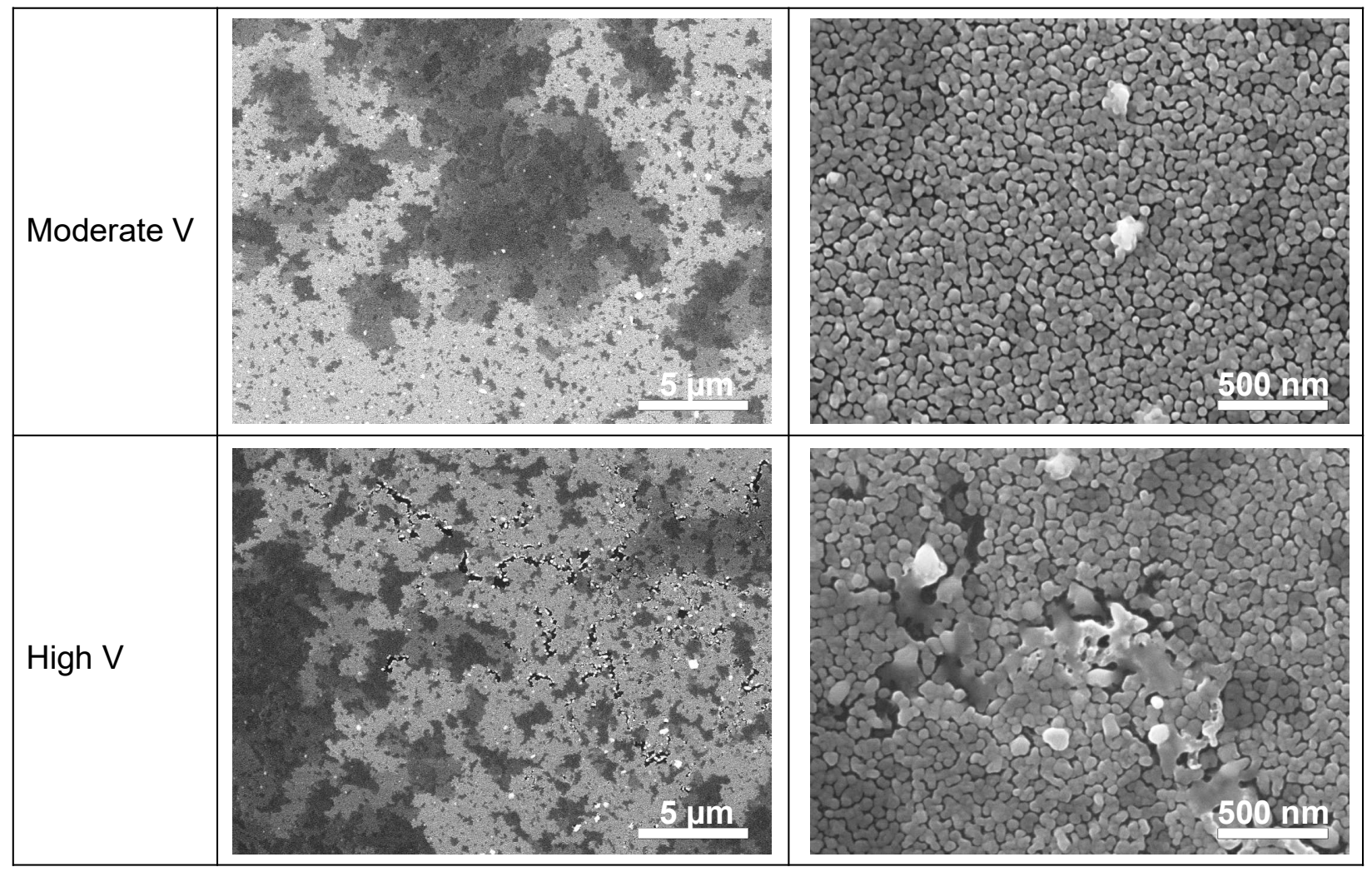

Figure S7: Scanning electron micrographs of the percolating network after application of moderate and high input signals (top and bottom rows, respectively). The left and right panels show the same films at different magnifications. At the moderate voltages used for our measurements the physical structure of the percolating network is unchanged. In contrast, application of high voltages causes obvious modifications of the physical structure. The nanoscale conducting filaments are similar to those observed in PENs. ${ }^{38}$ 


\section{References}

(1) Waldrop, M. M. The chips are down for Moore's law. Nature 2016, 530, 144-147.

(2) Markov, I. L. Limits on fundamental limits to computation. Nature 2014, 512, 147-154.

(3) Bullmore, E.; Sporns, O. The economy of brain network organization. Nature Reviews Neuroscience 2012, 13, 336-349.

(4) Wang, Z.; Wu, H.; Burr, G. W.; Hwang, C. S.; Wang, K. L.; Xia, Q.; Yang, J. J. Resistive switching materials for information processing. Nature Reviews Materials 2020, 5, 173195.

(5) Torrejon, J.; Riou, M.; Araujo, F. A.; Tsunegi, S.; Khalsa, G.; Querlioz, D.; Bortolotti, P.; Cros, V.; Yakushiji, K.; Fukushima, A.; Kubota, H.; Yuasa, S.; Stiles, M. D.; Grollier, J. Neuromorphic computing with nanoscale spintronic oscillators. Nature 2017, 547, 428-431.

(6) Merolla, P. A. et al. A million spiking-neuron integrated circuit with a scalable communication network and interface. Science 2014, 345, 668-673.

(7) Davies, M. et al. Loihi: A Neuromorphic Manycore Processor with On-Chip Learning. IEEE Micro 2018, 38, 82-99.

(8) Jo, S. H.; Chang, T.; Ebong, I.; Bhadviya, B. B.; Mazumder, P.; Lu, W. Nanoscale memristor device as synapse in neuromorphic systems. Nano Letters 2010, 10, 12971301.

(9) Burr, G. W. et al. Neuromorphic computing using non-volatile memory. Advances in Physics: X 2017, 2, 89-124.

(10) Wang, Z. et al. Fully memristive neural networks for pattern classification with unsupervised learning. Nature Electronics 2018, 1, 137-145. 
(11) Terabe, K.; Hasegawa, T.; Nakayama, T.; Aono, M. Quantized conductance atomic switch. Nature 2005, 433, 47-50.

(12) Stieg, A. Z.; Avizienis, A. V.; Sillin, H. O.; Martin-Olmos, C.; Aono, M.; Gimzewski, J. K. Emergent Criticality in Complex Turing B-Type Atomic Switch Networks. Advanced Materials 2012, 24, 286-293.

(13) Tuma, T.; Pantazi, A.; Le Gallo, M.; Sebastian, A.; Eleftheriou, E. Stochastic phasechange neurons. Nature Nanotechnology 2016, 11, 693-699.

(14) Srinivasa, N.; Stepp, N. D.; Cruz-Albrecht, J. Criticality as a Set-Point for Adaptive Behavior in Neuromorphic Hardware. Frontiers in Neuroscience 2015, 9, 449.

(15) Muñoz, M. A. Colloquium : Criticality and dynamical scaling in living systems. Reviews of Modern Physics 2018, 90, 031001.

(16) Deng, Z.; Zhang, Y. Collective Behavior of a Small-World Recurrent Neural System With Scale-Free Distribution. IEEE Transactions on Neural Networks 2007, 18, 13641375.

(17) Lukoševičius, M.; Jaeger, H. Reservoir computing approaches to recurrent neural network training. Computer Science Review 2009, 3, 127-149.

(18) Du, C.; Cai, F.; Zidan, M. A.; Ma, W.; Lee, S. H.; Lu, W. D. Reservoir computing using dynamic memristors for temporal information processing. Nature Communications 2017, 8, 2204.

(19) Bose, S. K.; Lawrence, C. P.; Liu, Z.; Makarenko, K. S.; van Damme, R. M. J.; Broersma, H. J.; van der Wiel, W. G. Evolution of a designless nanoparticle network into reconfigurable Boolean logic. Nature Nanotechnology 2015, 10, 1048-1052. 
(20) Cooper, A.; Zhong, C.; Kinoshita, Y.; Morrison, R. S.; Rolandi, M.; Zhang, M. Selfassembled chitin nanofiber templates for artificial neural networks. Journal of Materials Chemistry 2012, 22, 3105-3109.

(21) Milano, G.; Pedretti, G.; Fretto, M.; Boarino, L.; Benfenati, F.; Ielmini, D.; Valov, I.; Ricciardi, C. Self-organizing memristive nanowire networks with structural plasticity emulate biological neuronal circuits. Arxiv.Org 2019, arxiv.org/abs/1909.02438, Accessed 01-15-2020.

(22) Demis, E. C.; Aguilera, R.; Scharnhorst, K.; Aono, M.; Stieg, A. Z.; Gimzewski, J. K. Nanoarchitectonic atomic switch networks for unconventional computing. Japanese Journal of Applied Physics 2016, 55, 1102B2.

(23) Manning, H. G.; Niosi, F.; da Rocha, C. G.; Bellew, A. T.; O'Callaghan, C.; Biswas, S.; Flowers, P. F.; Wiley, B. J.; Holmes, J. D.; Ferreira, M. S.; Boland, J. J. Emergence of winner-takes-all connectivity paths in random nanowire networks. Nature Communications 2018, 9, 3219 .

(24) Tanaka, H.; Akai-Kasaya, M.; TermehYousefi, A.; Hong, L.; Fu, L.; Tamukoh, H.; Tanaka, D.; Asai, T.; Ogawa, T. A molecular neuromorphic network device consisting of single-walled carbon nanotubes complexed with polyoxometalate. Nature Communications 2018, 9, 2693.

(25) Sattar, A.; Fostner, S.; Brown, S. A. Quantized Conductance and Switching in Percolating Nanoparticle Films. Physical Review Letters 2013, 111, 136808.

(26) Bose, S. K.; Mallinson, J. B.; Gazoni, R. M.; Brown, S. A. Stable self-assembled atomicswitch networks for neuromorphic applications. IEEE Transactions on Electron Devices 2017, 64, 5194-5201.

(27) Bose, S. K.; Shirai, S.; Mallinson, J. B.; Brown, S. A. Synaptic dynamics in complex self-assembled nanoparticle networks. Faraday Discussions 2019, 213, 471-485. 
(28) Minnai, C.; Bellacicca, A.; Brown, S. A.; Milani, P. Facile fabrication of complex networks of memristive devices. Scientific Reports 2017, 7, 7955.

(29) Mallinson, J. B.; Shirai, S.; Acharya, S. K.; Bose, S. K.; Galli, E.; Brown, S. A. Avalanches and criticality in self-organized nanoscale networks. Science Advances 2019, 5, eaaw8438.

(30) Shirai, S.; Acharya, S. K.; Bose, S. K.; Mallinson, J. B.; Galli, E.; Pike, M. D.; Arnold, M. D.; Brown, S. A. Long-range temporal correlations in scale-free neuromorphic networks. Network Neuroscience 2020, 4, 432-447.

(31) Friedman, N.; Ito, S.; Brinkman, B. A. W.; Shimono, M.; DeVille, R. E. L.; Dahmen, K. A.; Beggs, J. M.; Butler, T. C. Universal Critical Dynamics in High Resolution Neuronal Avalanche Data. Physical Review Letters 2012, 108, 208102.

(32) Bak, P.; Tang, C.; Wiesenfeld, K. Self-organized criticality. Physical Review A 1988, $38,364-374$.

(33) Olsen, M.; Hummelgård, M.; Olin, H. Surface Modifications by Field Induced Diffusion. PLoS ONE 2012, 7, e30106.

(34) Onofrio, N.; Guzman, D.; Strachan, A. Atomic origin of ultrafast resistance switching in nanoscale electrometallization cells. Nature Materials 2015, 14, 440-446.

(35) Xiang, C.; Kim, J. Y.; Penner, R. M. Reconnectable Sub-5 nm Nanogaps in Ultralong Gold Nanowires. Nano Letters 2009, 9, 2133-2138.

(36) Stauffer, D.; Aharony, A. Introduction to Percolation Theory; Taylor \& Francis, 2003.

(37) Fostner, S.; Brown, R.; Carr, J.; Brown, S. A. Continuum percolation with tunneling. Physical Review B 2014, 89, 075402.

(38) Yang, Y.; Chen, B.; Lu, W. D. Memristive Physically Evolving Networks Enabling the Emulation of Heterosynaptic Plasticity. Advanced Materials 2015, 27, 7720-7727. 
(39) Shew, W. L.; Plenz, D. The functional benefits of criticality in the cortex. Neuroscientist 2013, 19, 88-100.

(40) Barnett, R. N.; Landman, U. Cluster-derived structures and conductance fluctuations in nanowires. Nature 1997, 387, 788-791.

(41) Wang, W.; Wang, M.; Ambrosi, E.; Bricalli, A.; Laudato, M.; Sun, Z.; Chen, X.; Ielmini, D. Surface diffusion-limited lifetime of silver and copper nanofilaments in resistive switching devices. Nature Communications 2019, 10, 81.

(42) Burkitt, A. N. A Review of the Integrate-and-fire Neuron Model: I. Homogeneous Synaptic Input. Biological Cybernetics 2006, 95, 1-19.

(43) Gerstner, W.; Kistler, W. M.; Naud, R.; Paninski, L. Neuronal Dynamics; Cambridge University Press: Cambridge, 2014.

(44) Sethna, J. P.; Dahmen, K. A.; Myers, C. R. Crackling noise. Nature 2001, 410, 242-250.

(45) Grimaldi, C. Theory of percolation and tunneling regimes in nanogranular metal films. Physical Review B 2014, 89, 214201.

(46) Fostner, S.; Brown, S. A. Neuromorphic behavior in percolating nanoparticle films. Physical Review E 2015, 92, 052134.

(47) Rubinov, M.; Sporns, O.; Thivierge, J.-P.; Breakspear, M. Neurobiologically Realistic Determinants of Self-Organized Criticality in Networks of Spiking Neurons. PLoS Computational Biology 2011, 7, e1002038.

(48) Denève, S.; Machens, C. K. Efficient codes and balanced networks. Nature Neuroscience 2016, 19, 375-382.

(49) Schmelzer, J.; Brown, S. A.; Wurl, A.; Hyslop, M.; Blaikie, R. J. Finite-Size Effects in the Conductivity of Cluster Assembled Nanostructures. Physical Review Letters 2002, 88,226802 . 
(50) Clauset, A.; Shalizi, C. R.; Newman, M. E. J. Power-law distributions in empirical data. SIAM Review 2009, 51, 661-703.

(51) Deluca, A.; Corral, Á. Fitting and goodness-of-fit test of non-truncated and truncated power-law distributions. Acta Geophysica 2013, 61, 1351-1394.

(52) Wagenmakers, E.-J.; Farrell, S. AIC model selection using Akaike weights. Psychonomic Bulletin \& Review 2004, 11, 192-196. 


\section{${ }_{447}$ Graphical TOC Entry}

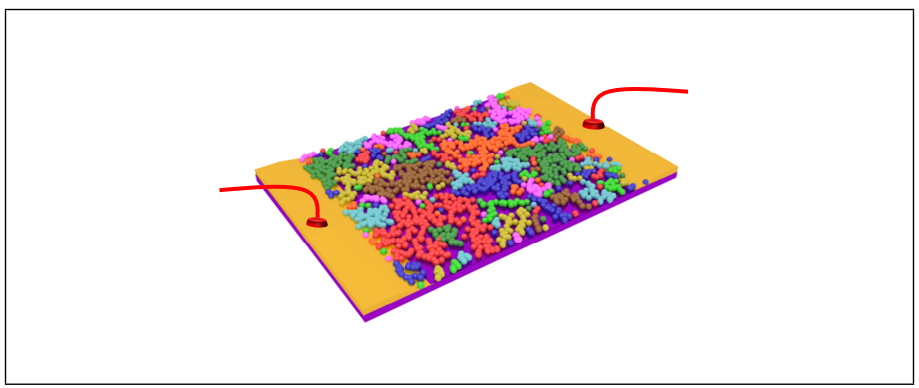

Schematic of percolating nanoparticle network. 